\title{
Impacts of Renewable Sources on Power Quality in Distribution Systems
}

\author{
Josep Balcells ${ }^{1}$, Jaroslav Doležal ${ }^{2}$, Josef Tlustý², Viktor Valouch ${ }^{3}$ \\ ${ }^{1}$ Electronics Engineering Dept. of Universitat Politècnica de Catalunya, Campus de Terrassa (Spain) \\ E-mail: balcells@eel.upc.es \\ ${ }^{2}$ Department of Power Engineering, Czech Technical University in Prague \\ Technicka 2, 16627 Prague 6 (Czech Republic) \\ E-mail: dolezalj@feld.cvut.cz, tlusty@feld.cvut.cz \\ ${ }^{3}$ Institute of Electrical Engineering of the Academy of Sciences of the Czech Republic \\ Dolejškova 5, 18202 Prague 8 (Czech Republic) \\ E-mail: valouch@iee.cas.cz
}

\begin{abstract}
This paper analyzes the influence of renewable sources (RS) on voltage quality in MV networks and summarizes problems considered of general interest relating to the power quality of dispersed generation. We are interested in voltage fluctuations, flicker, harmonic distortion, interharmonic distortion, and the effect of dispersed generation on ripple control signals. The influence of DG on voltage stabilization, line losses, and short circuit impedance are also investigated. A basic analysis of the possibility and effectiveness of using a parallel Active Filter (AF) to compensate for the constant-speed wind turbine (WT) flicker, reactive power, and terminal voltage variation is presented too.
\end{abstract}

Keywords: Dispersed generation, flicker, harmonics, ripple control, voltage quality, voltage fluctuation, active filters

\section{Introduction}

Operations of renewable sources within MV or LV can produce different types of disturbance in distribution networks. Voltage quality can be improved by finding optimal points of connection with the network to minimize the inverse impact of the connection of dispersed generation to Medium Voltage distribution systems.

To investigate the phenomena of system perturbations from dispersed generation (DG), it is often necessary to make rough calculations in distribution networks.

For a complex appreciation of the small energy sources within distribution networks a number of Czech standards (PNE 333430 - Parameters of power quality) were established.

The standard PNE 33 3430-0 deals with computational evaluation of inverse-impact non-linear loads on distribution networks. Following standards of this rank from PNE 33 3430-1 to 33 3430-6 covers the compatibility levels for voltage fluctuations, imbalance and harmonics. They also define the measuring and assessment methods and the required measuring accuracy in distribution systems. These standards deal with the connection of dispersed generation and equipment with non-linear characteristics or flexible operational characteristics.

Parameters of power quality are also the subject of international standards and recommendations (IEC rank 1000, EN rank 61 000). The standard PNE rank 333430 reflect the current state of this subject in the West European countries as well.

In line with existing standards, "The rules for parallel operation of the dispersed sources with distribution companies' network LV or MV," were prepared. This document is a part of "Rules for the operation of distribution systems." (Codex).

\section{Standpoint for the Connecting of Loads and Small Energy Sources}

According to PNE 33 3430-0 "Computational evaluation of the inverse impact of distribution systems customers" we have to check following impacts:

- voltage variations and fluctuations,

- imbalances,

- harmonics and interharmonics,

- impacts on ripple control.

Furthermore we must check all conditions assuring the safe connection of small energy sources within networks, their operation and disconnection in extraordinary states (for example in the case of voltage loss in the distribution network, or islanding operation.).

\section{A. Voltage Variations and Fluctuations}

The coefficient of flicker severity $A_{\mathrm{lt}}\left(P_{\mathrm{lt}}\right)$ presents a criterion to evaluate the voltage variations and fluctuations due to small power sources. For real power systems, this coefficient can be measured or calculated. Coefficient $A_{\mathrm{lt}}$ depends on a short circuit current at a given 
point, nominal generation power and the flicker coefficient of equipment $c[1]$.

Coefficient $c$ indicates the capability of equipment to produce flicker, and characterizes equability of operation of small power plants. With regard to flicker, the generators driven by gas or stream turbines are positively evaluated (coefficient $c$ has been less than 20)

Generators of wind power plants are, with regard to flicker, evaluated very negatively because their flicker coefficient is approximately 40. Customer loading changes cause voltage variations in distribution networks, what can have a periodic or random character, (sources of voltage variations and fluctuations can be electrical arc furnaces, welding equipments, and starting asynchronous motors, etc.). Admissible levels for rectangular voltage fluctuations depend on their frequency and are given by curves mentioned in standard PNE 33 3430-0. This curve lay down the limiting magnitude of short-time flicker severity $P_{\mathrm{st}}=1$. For voltage variations of the small sources connecting within MV the limit $2 \%$ is used. A long-time flicker severity caused by operation of small sources is limited by value $P_{\mathrm{lt}}=0.46$. The check must above all be performed on wind power plants.

\section{B. Inverse Impact on Ripple Control}

An audio frequency signal is superimposed on a supply waveform as a short sequence of impulses, forming a code format for transmitted control orders. This system enables remote on or off switching of different loads. For the remote control system to function effectively, the following conditions must be fulfilled:

- the audio frequency signal must have a voltage overlapping functional voltage of receivers,

- customers' equipment must not decrease the level of control impulse voltage and must not emit disturbing signals to distribution systems,

- customers' equipment must not exceed the load of the ripple control transmitter.

Ripple control systems work with low frequency, generally around $200 \mathrm{~Hz}$, and the impulse voltage level is between 1.8 and $4 \% U_{\mathrm{n}}$.

The minimum accepted value of load impedance at the point of common coupling (PCC) is given by the help of impedance coefficient $\alpha$ (expressive accepted minimum value of load impedance on ripple control frequency in reference to connecting impedance on fundamental frequency) for equipment connecting within the MV network. Dispersed generation can have a negative impact on ripple control voltage due to low DG impedance at the PCC.

\section{Harmonics and Interharmonics}

In medium-voltage networks, harmonic voltages can arise from the influence of equipment attached to the low-, medium-, or high-voltage networks [1]. For this reason, any given piece of equipment can only produce part of any compatible harmonic voltage level. In the Czech Republic, there are special conditions for harmonics and interharmonics given in the regulations for the connection of DG to networks. When planning new DG installations, special attention must be paid to the potential effect on the network of harmonic and interharmonic voltage levels generated by the installation [1].

\section{Case Study of DG Connecting within MV}

Last year new applications of 5 MW total dispersed generation connected within $22 \mathrm{kV}$ network was solved. Points of common coupling of DGs with the MV network were relatively far from the $110 / 22 \mathrm{kV}$ supply substation, and the DGs power was high considering the overhead power line cross-section. Some specialists from the Power Distribution Company have been afraid of voltage variation and harmonics in the network due to DG operation. For this reason a case study of the negative impact of DG on network was made [2]. The study deals with load flow, losses, and harmonics in the MV network with and without DG. The scheme of the $22 \mathrm{kV}$ network is in following figure.

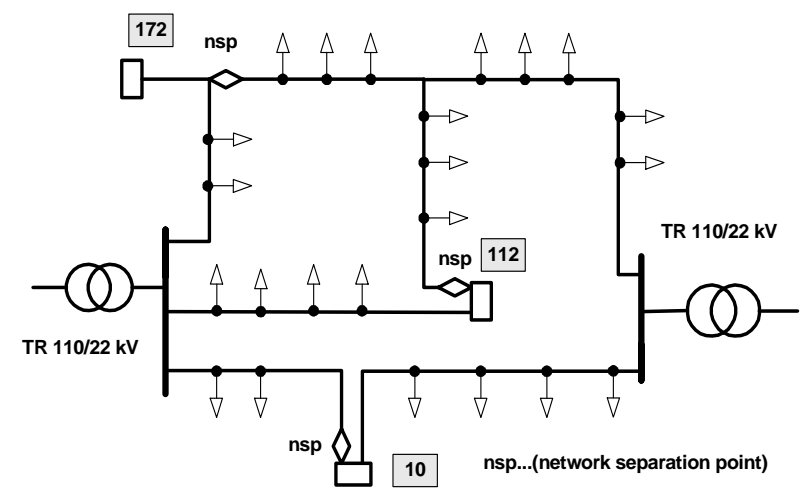

Fig. 1. Sample $22 \mathrm{kV}$ network with DG installation

The MV network has 255 points and around 190 branches. The industrial sources of disturbance are in points 112 (converter $1 \mathrm{MW}$ ), and 172 (converter 400 $\mathrm{kW}$ ). DG is installed in point 10 . Usually the mesh network is disconnected at separation points, and for conventional operation the radial network is used.

\section{A. Voltage Profile Calculations}

The load-flow calculation for the distribution network was made. The calculations give rise to the following facts. Network voltage in PCC without DG is $22.6 \mathrm{kV}$. If DG with the power factor 0.95 is connected to network, the voltage in PCC will reach a value of $23.5 \mathrm{kV}$ (voltage increasing by over 2.6\%). Calculations show that the most applicable way of DG operation is with a power factor equal to one; in this case voltage variation in the network is less than $2 \%$.

\section{B. DG Operation Impact on MV System Losses}

The power losses in the $22 \mathrm{kV}$ MV network with DG operation decreased. At the same time, the calculation 
showed that if the demand on limiting voltage variation less $2 \%$ were fulfilled (DG works with a power factor equal to one), then the losses were higher than during a DG operation with a power factor equal to 0.95 . The best operation of DG from the aspects of voltage variations and power losses is given by a power factor between 0.95 and 1.

\section{DG Operation Impact on Harmonic Voltage}

An analysis of harmonic voltage in the real $22 \mathrm{kV}$ network was made. Non-linear loads (industrial drives with converters) were simulated by harmonic current sources, power lines by longitudinal and transversal parameters (R, L, G, C), step-down transformers 22/0.4 $\mathrm{kV}$ by longitudinal inductance, $\mathrm{LV}$ loads were simulated by CIGRE model assuming $50 \%$ of the nominal transformer load.

Calculation for compartmental network to radial parts for three basic cases was made:

- $\quad$ non-loaded step-down transformers $22 / 0.4 \mathrm{kV}$ with DG,

- $50 \%$ loaded step-down transformers without DG,

- $50 \%$ loaded step-down transformers with DG.

Harmonic voltage levels were compared with the standard PNE 33 3430-1, where compatibility levels for MV distribution networks were given.

The calculation results of harmonic voltages when MV/LV distribution transformers are 50\% loaded and DG is connected to the network are higher than the compatibility level at bus 172 only for higher harmonics. The calculated values of harmonic voltages with standard compatible levels are given in the next figure.

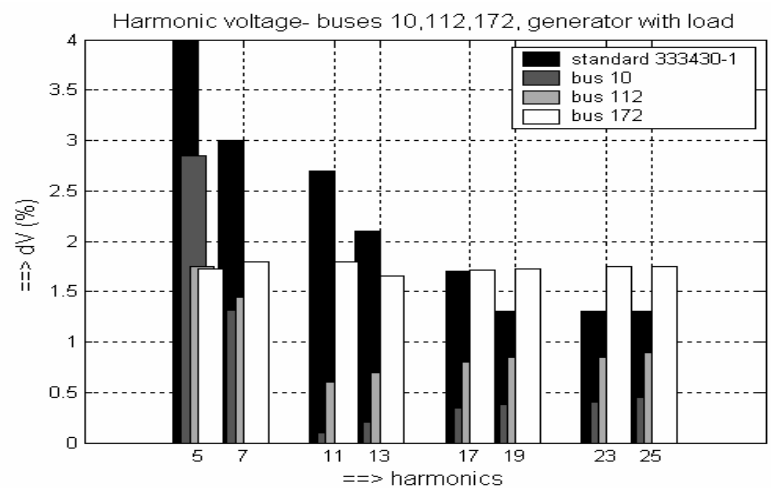

Fig. 2. Harmonic voltage level in important buses

Because of the high level of harmonics in bus 172, a special filter is installed that has two branches tuned to the $11^{\text {th }}$ and $17^{\text {th }}$ harmonic in order to decrease harmonic voltage at higher frequencies. The following graphs show the effect the filter has on the distribution of frequencies.

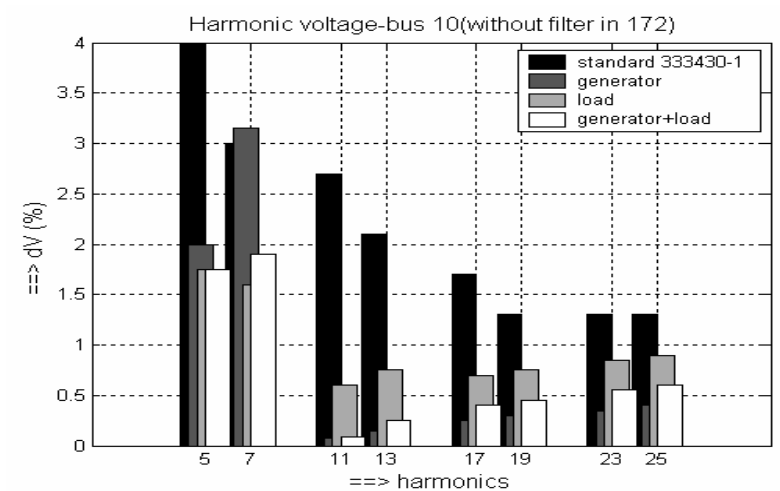

Fig. 3. Harmonic voltage level at bus 10 without filter

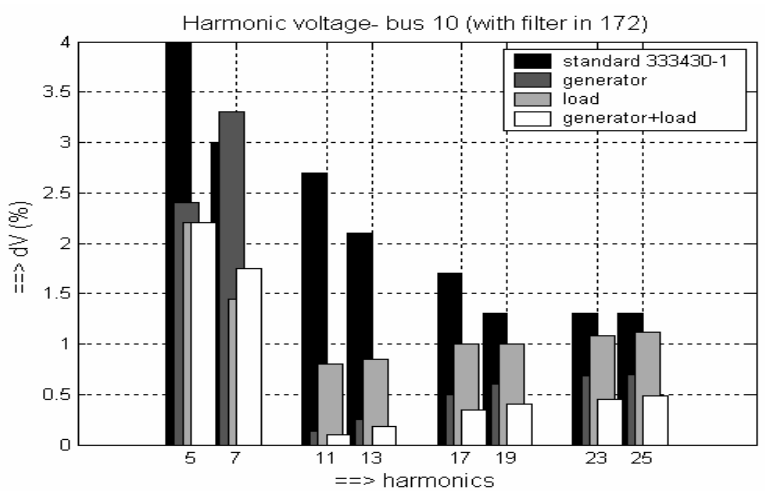

Fig. 4. Harmonic voltage level at bus 10 with filter

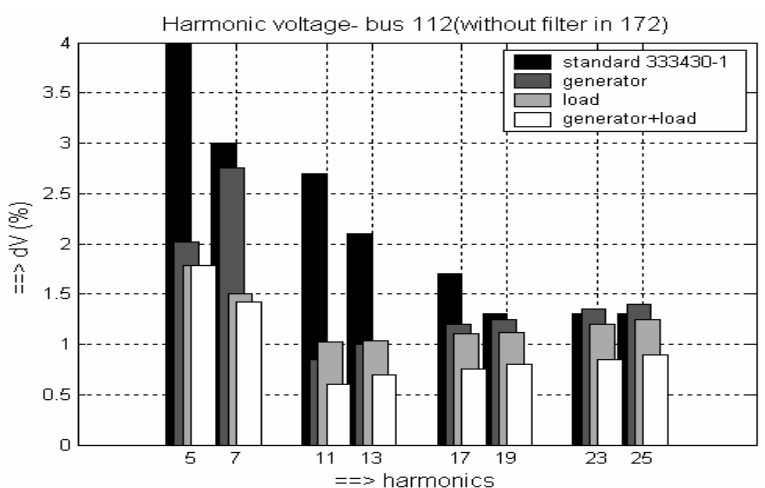

Fig. 5. Harmonic voltage level at bus 112 without filter

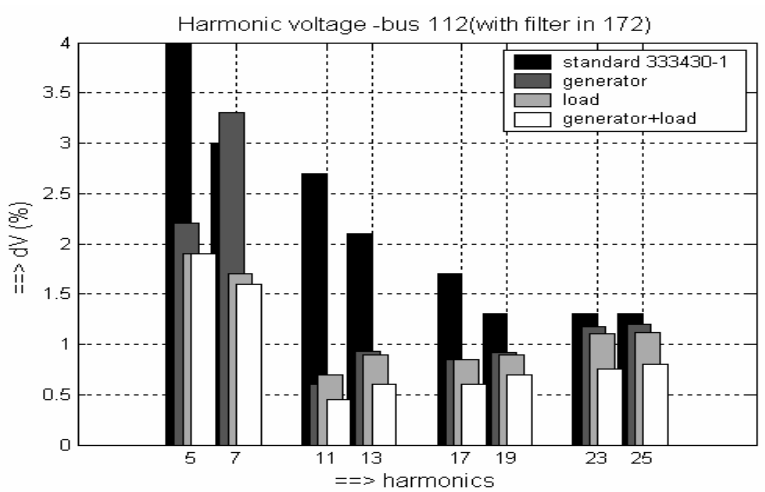

Fig. 6. Harmonic voltage level at bus 112 with filter 


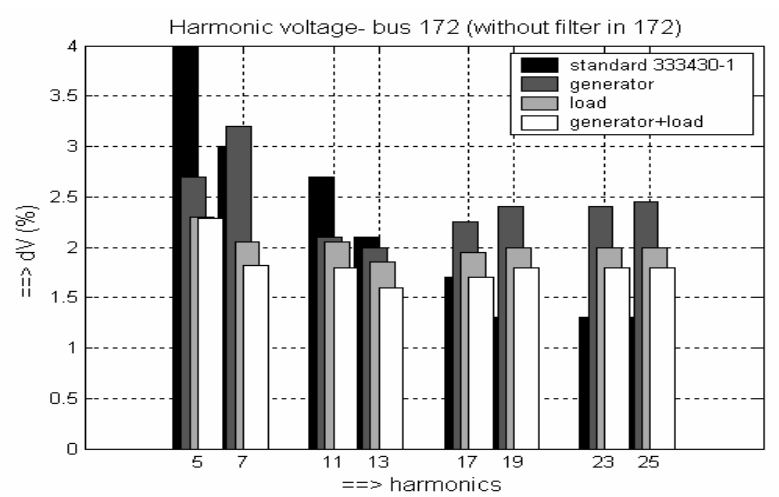

Fig. 7. Harmonic voltage level at bus 172 without filter

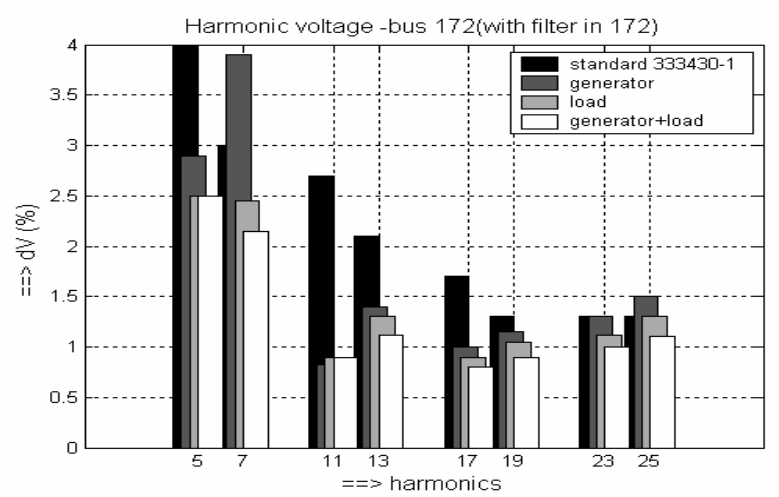

Fig. 8. Harmonic voltage level at bus 172 with filter

The graphs indicate that the connection of DG in this case not only causes no abnormal disruption of the network but actually improves network performance.

\section{DG Impact on Ripple Control}

The calculation of the flow of ripple-control signals showed that there was no significant attenuation of the signal due to the connection of DG and the installation of filters.

Ripple signal level at points 10,112 , and 172 for connected generation, filters and transformer loads (for the nominal voltage level $2 \%$ at the HV network supply point) are changed from $1.77 \%$ to $2.12 \%$.

\section{Case Study of WT Flicker Compensation by Means of Active Filter}

The flicker, belonging among important power quality phenomena, is caused by power fluctuations generated by WT. The power fluctuation is produced by both the tower shadow effect and the wind gradient. The frequency of the power fluctuation corresponds to the WT rotor speed multiplied by the number of blades and it is usually about 1 or $1.5 \mathrm{~Hz}$. The most common constant-speed WTs are equipped with an induction generator connected directly to the grid. The pulsating torque of the induction generator, which is caused by the effects mentioned above, results in reactive and mainly active power fluctuations. The respective WT current fluctuation leads to a fluctuating voltage drop on the grid impedance, so the WT terminal voltage fluctuates too. These phenomena, namely the flicker and the voltage variation, do not occur practically at variable-speed WT, equipped with power electronic converters (PEC) that are able to suppress such parasitic effects.

There is a group of power electronics-based compensation devices used in Flexible AC Transmission System (FACTS) and Custom Power (CP) concepts. This technology offers many compensation functions for power transmission and distribution systems and represents the most progressive way to increase power quality in the systems, e.g. [3].

In the following, a basic analysis of the possibility and effectiveness of using a parallel Active Filter (AF) to compensate for the constant-speed WT flicker, reactive power and terminal voltage variation will be presented.

Fig. 9 shows a possible equivalent circuit of the parallel AF implementation into a power system with the WT. The parallel AF can be viewed as a current source. The scheme is simplified to make a basic analysis easier.

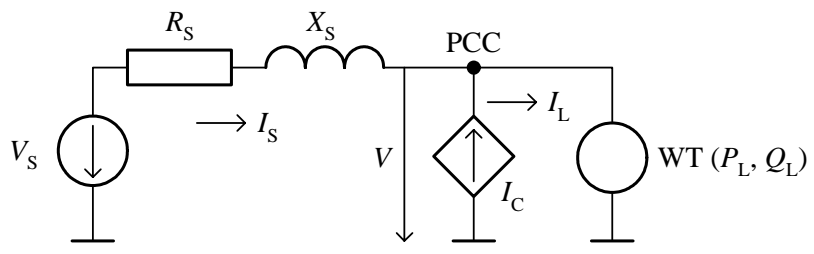

Fig. 9. AC power system with WT and parallel AF

The fluctuating voltage is generated by the fluctuating generator slip frequency, which is induced by the changing of a WT driven torque. The aim of the AF is to compensate for the voltage $V$ fluctuation to hold the constant voltage at the Point of Common Coupling (PCC).

In [4], the condition for attaining $|\boldsymbol{V}|=\left|\boldsymbol{V}_{\mathrm{S}}\right|$ was presented if the parallel AF is used. If we put the vector $V(=V)$ into the line of the axis $d$ of the reference frame rotating with the fundamental frequency $\omega_{1}$, the condition reads

$$
I_{\mathrm{Sq}}=\frac{R_{\mathrm{S}}}{X_{\mathrm{S}}} I_{\mathrm{Sd}}+\frac{3 V_{\mathrm{S}}}{2 S_{\mathrm{CC}}} I_{\mathrm{Sd}}^{2}
$$

where $V_{\mathrm{S}}=\left|V_{\mathrm{S}}\right|$ and $S_{\mathrm{CC}}=3 V_{\mathrm{S}}^{2} / Z_{\mathrm{S}}$ is the short circuit power at the PCC. The condition (1) holds for steady-states and we will consider the symmetrical system in the following analysis.

Let the active and reactive power of the WT are $P_{\mathrm{L}}$ and $Q_{\mathrm{L}}$. If the current $I_{\mathrm{C}}$ can be injected into the PCC in any position towards the voltage $V$, that is both the active $P_{\mathrm{AFP}}$ and reactive $Q_{\mathrm{AFP}}$ power may be generated by this shunt AF, we have two degree of freedom to control the power flow at the PCC. The first degree may be used to fulfil the condition (1), while the second one for the control of the amount of the active $P_{\mathrm{L}}$ power withdrawn from or delivered to the grid. 
Fig. 10 shows block diagram of the control system of the parallel AF. The compensation of fluctuating (subharmonic) components of active and reactive powers uses the concept of the Instantaneous Reactive Power (IRP) theory [5]. The fluctuating power components may be obtained by using appropriate Signal Filters (SF) with cut-off frequency $\omega_{\mathrm{c}}$. The coefficient $k_{\mathrm{dp}}$ determines whether the fluctuating active power should be compensated for $\left(k_{\mathrm{dp}}=1\right)$ or not $\left(k_{\mathrm{dp}}=0\right)$ by the parallel AF. The control strategy of the parallel AF involves also the algorithm

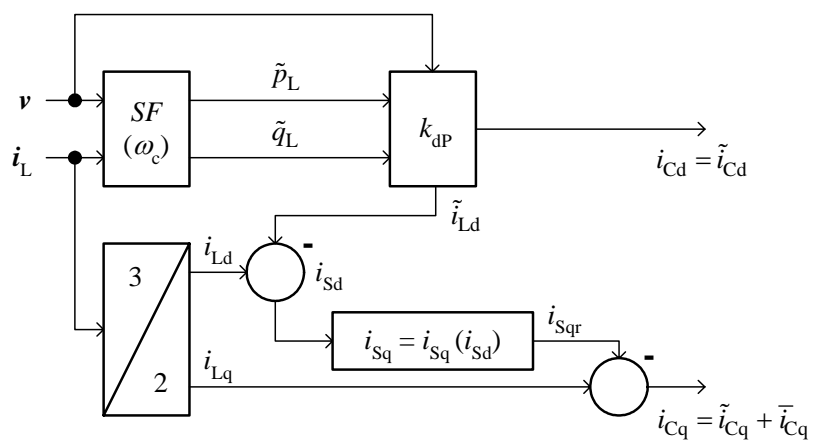

Fig. 10. Block diagram of the control system of parallel AF

for attaining $|\boldsymbol{V}|=\left|\boldsymbol{V}_{\mathrm{S}}\right|$ in steady states, which has been described before. The variable $i_{\text {Sqr }}$ represents the reference $q$-component of the current $i_{\mathrm{S}}$ of the supply system, demanded for fulfilling the previous condition for basic voltage harmonics. A dc capacitor voltage feedback control loop is not included in the figure.

For the analysis of the control system and synthesis of its parameters the frequency domain approach or transfer functions are usually used. The vectors characterising variables of three phase systems in transients are mostly applied. The basic assumption for using the methods mentioned is the linearity of the system under analysis.

Based on the analysis of the power circuit in Fig. 9, the control structure in Fig. 10 and on the equation (1), a linearised model of the system may be developed in the $s$-domain or the frequency domain. Except the equation (1), other equations describing the power and control circuit (including specific signal filters) are linear so they hold their forms regardless the absolute or perturbed signals are in question. The model makes it possible to analyse basic properties of the system for perturbed signals and different control topologies and parameters.

The magnitude $I_{\mathrm{L}}$ of the simulated phase current of the WT generator with the fundamental frequency $\omega_{1}$ was set as nominal one and the magnitude of the fluctuation of the induction generator slip frequency was chosen in such a way to induce the current fluctuation of $\pm 25 \%$ of the current nominal value. The frequency of the fluctuation is $\omega_{\mathrm{k}}=1.5 \mathrm{~Hz}$ (0.03 in the p.u. system).

Fig. 11 presents amplitude frequency characteristics showing the magnitude of fluctuating apparent power of the parallel $\mathrm{AF}$, demanded for the mitigation of the voltage fluctuation, and the magnitude $d_{v}$ of this residual voltage fluctuation at the PCC. The magnitude $d_{v}=0.013$ p.u. was calculated by using the same model when there is no compensation. The rated power of the AF belongs among main criteria showing how effective the compensation strategy is and determines actually the feasibility of a project [6]. The linearised model of the system with the parameters $R_{\mathrm{S}}=0.005, L_{\mathrm{S}}=0.05, V_{\mathrm{S}}=1, P_{\mathrm{L}}=1$ (all in p.u.), power factor $=0.95$ was used, supposing that the WT generator works at its rated mode of operation and the current fluctuation is $\pm 25 \%$ of the current nominal value. The signal filters of the first order with the cut-off frequency $\omega_{\mathrm{c}}=0.005$ p.u. were used for filtering WT power components. According to Fig. 10 the parallel AF compensates the whole reactive power consumed by the WT, delivers to the network a reactive power to hold the voltage at the PCC constant, and compensates the fluctuating active power of the WT $\left(k_{\mathrm{dP}}=1\right)$. In fact, the linearised model-based analysis in the frequency domain considers only the flow of fluctuating components, so if we want to compensate for also the voltage drop at the PCC and reactive power of the WT generator, the rated power of the real parallel AF must be high enough to fulfil these demands.

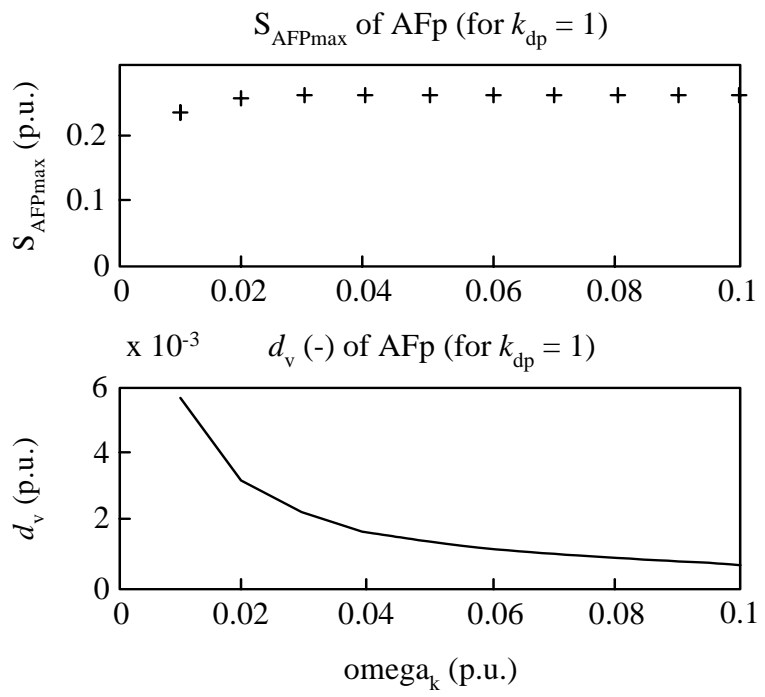

Fig. 11. Demanded power of parallel AF for mitigation of the voltage subharmonic component at PCC and magnitude of this residual voltage versus frequency of the flicker

Figs. 12 and 13 show time responses of main variables of the system in steady states for the flicker frequency 1.5 $\mathrm{Hz}$. The responses have been obtained by simulation of the nonlinear model.

Fig. 12 presents the time responses of the grid current components, the difference between the voltage at the PCC and the source voltage, the load current components, and the torque of the induction generator when the $\mathrm{AF}$ is not in the operation. 


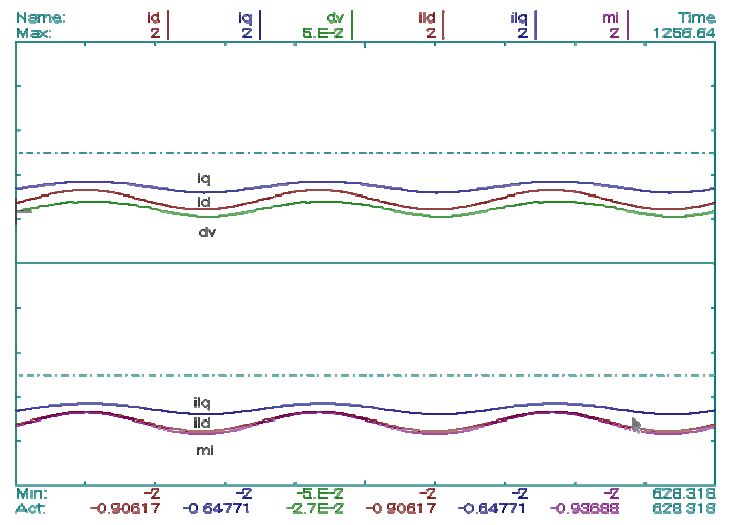

Fig. 12. Time responses of main variables of the system in steady states for the flicker frequency $1.5 \mathrm{~Hz}$ obtained by the simulation of the nonlinear model. AF is not in the operation

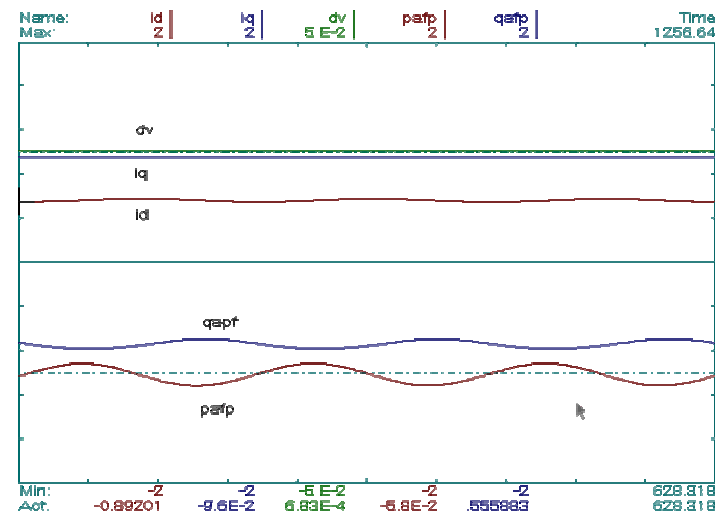

Fig. 13. Time responses of main variables of the system in steady states for the flicker frequency $1.5 \mathrm{~Hz}$ obtained by the simulation of the nonlinear model. AF compensates the whole reactive power and the fluctuating component of the active power

Fig. 13 shows again the responses of the grid current components, the difference between the voltage at the PCC and the source voltage, and instantaneous active and non-active power components of the parallel AF after introducing the $\mathrm{AF}$ in the function. The $\mathrm{AF}$ compensates for practically the whole reactive power and the fluctuating component of the active power. In fact, some other options with low AF power demands can be chosen (e.g. the compensation of the fluctuating active and/or reactive power only) in accordance with the selected modification of the presented control strategy of the AF.

The dependences of the magnitudes of the residual voltage fluctuation and the pulsating apparent power of the $\mathrm{AF}$ on the flicker frequency, predicted in Fig. 11 for the linearised model, have been confirmed by the nonlinear simulation.

\section{Conclusions}

The first case study shows that it is very important to calculate beforehand the state of the system and to model the impact that harmonic and interharmonic voltages from DG equipment might have upon it. It is important not to forget to check the value of ripple-control signals in the network, especially when filters are to be used to control harmonics. When the DG goes into operation, it is important to confirm pre-operation modelling with measurement of actual operation. In our case, there was an excellent correspondence between predicted and actual values.

As for the second case study, we can come to the conclusion that the parallel AF is effective enough to substantially improve the voltage fluctuations with frequencies even below $2 \mathrm{~Hz}$, which is the case of the flicker at constant-speed WTs. The presented analysis indicates that from the point of view of the voltage stabilization and flicker mitigation the most effective may be the compensation of the whole reactive power as well as the fluctuating component of the active power of the WT. In fact, this strategy needs higher rated power (or current) of the AF then simpler control modifications. Nevertheless, this power is still substantially lower then the WT power. Contrary to that, the PECs used at the variable-speed WT, which feature similar capabilities, must be designed for full WT powers that are transferred through them.

\section{Acknowledgement}

The financial supports of the Grant Agency of the Czech Republic (project No. 102/03/1551) and the Grant Agency of the Academy of the Sciences of the Czech Republic (project No. A2057301) are highly acknowledged.

\section{References}

[1] J. Doležal and J. Tlustý, "Interharmonics, Voltage Fluctuation, and Flicker in Supply Power Systems”, in Proc. PEMC '98, Prague, pp. 6/100-6/103.

[2] J. Svab, "Connecting of Dispersed Generation with Distribution Networks”, Tech. Rep., CTU Prague, Nov. 2000.

[3] H. Fugita and H. Akagi, "The Unified Power Quality conditioner. The Integration of Series-and Shunt-Active Filters,” IEEE Trans. On PE-13, No. 2, March 1998, 315322.

[4] G. De Préville, "Flicker Mitigation, Application to a STATCOM,” EPE 2001, Graz, Austria, September 2001, CD-ROM.

[5] H. Akagi, "New Trends in Active Filters for Power Conditioning," IEEE Transactions on IA-32, No. 6, 1996, 1312-1322.

[6] D. D. Bester, A. D. Le Roux, H. T. Monston and J. H. R. Enslin, "Evaluation of Power-Ratings for Active Power Quality Compensators,” EPE 1999, Lausanne, Switzerland, 1999, CD-ROM. 